\title{
Dynamic hologram magnification with incoherent optical pumping
}

Article in Proceedings of SPIE - The International Society for Optical Engineering · July 2003

DOI: $10.1117 / 12.498464$

CITATION

1

2 authors:

\section{Omar Ormachea}

Universidad Privada Boliviana

54 PUBLICATIONS 86 CITATIONS

SEE PROFILE
READS

12
Alexei Tolstik

Belarusian State University

151 PUBLICATIONS 356 CITATIONS

SEE PROFILE 


\title{
PROCEEDINGS OF SPIE
}

SPIE-The International Society for Optical Engineering

\section{Photonics, Devices, and Systems II}

\author{
Miroslav Hrabovský \\ Dagmar Senderáková \\ Pavel Tománek \\ Editors
}

Proceedings from PHOTONICS PRAGUE 2002

26-29 May 2002

Prague, Czech Republic

Organized by

CSSF-Czech and Slovak Society for Photonics

Tech-Market (Czech Republic)

Sponsored by

EOARD-European Office of Aerospace Research and Development,

U.S. Air Force Research Laboratory

EOS-European Optical Society

SPIE-The International Society for Optical Engineering Czech/Slovak Chapter

ICO-The International Commission for Optics

Czech Technical University in Prague

Academy of Sciences of the Czech Republic

Slovak Academy of Sciences

Published by

SPIE-The International Society for Optical Engineering

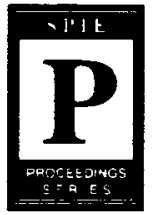

Volume 5036

SPIE is an international technical society dedicated to advancing engineering and scientific applications of optical, photonic, imaging, electronic, and optoelectronic technologies. 


\title{
Dynamic hologram magnification with incoherent optical pumping
}

\author{
Omar Ormachea, Alexei L. Tolstik \\ Belarusian State University, Minsk
}

\begin{abstract}
In the present work consideration is given to theoretical and experimental analysis of the possibilities for improving the efficiency of multiwave mixing in the process of dynamic hologram recording in solutions of complex organic compounds with additional irradiation of resonant medium at a frequency falling within the absorption band from the excited level. Three- and seven-fold increase in the diffraction efficiency has been realized in Rhodamine $6 \mathrm{G}$ dye for four- and six-wave mixing, respectively.
\end{abstract}

Keywords: Multiwave mixing, dynamic holograms, high order nonlinearities

\section{INTRODUCTION}

Active investigations of multiwave mixing realized in media with the fifth- and higher-order nonlinearities offer the scope for wider nonlinear spectroscopy methods and diffraction methods providing transformation and control of the laser beam spatial structure. Such media are associated with the holographic groove profile distortions traditionally considered as a negative factor resulting in noise components of the diffracted radiation. At the same time, new components occurring in Fourier decomposition of the medium nonlinear susceptibility into space harmonics of a dynamic grating enable Bragg diffraction of a reading beam to be realized into the second or higher orders ${ }^{1-3}$. An analysis of the properties of nonlinear holographic elements was conductive to the development of methods for optical information processing (spatial filtering, associative memory) and made it possible to attain various transformations of light beams (wavefront profile transformation, variations in propagation direction, deformation corrections for the space structure, frequency conversion of images, etc.). In nonlinear optics the processes due to higher order nonlinearities are of particular interest allow realization of the signals otherwise impossible in experiments with nonlinearities of lower orders. Through the use of multiwave mixing one is enabled to measure directly the fifth- and higher-order nonlinear optical susceptibilities, to isolate the contributions of different nonlinearities, relaxation mechanisms and dephasing, to improve the potentialities of the Doppler-free spectroscopy method. Transition to the femtosecond range makes possible the real time studies into the dynamic properties of local structures in liquid substances and into the superfast molecular processes, including electronic transition dephasing, nuclear mobility in liquids, population of upper excited states. Applicability of multiwave processes is however hindered by little values of higher-order nonlinearities and hence low efficiency of multiwave mixing.

This work presents a new method for increasing the energy efficiency of multiwave mixing in solutions of complex organic compounds based on the dynamic hologram magnification on absorption of incoherent external radiation by the molecules in the excited states. Theoretical substantiation has been performed on the basis of a three-level resonant medium model in the assumption that the frequency of the wave involved in the process of multiwave mixing falls within the absorption band from the ground level, whereas the frequency of an additional light beam (optical pumping) is tuned out into the absorption band from the excited level. The experimental results presented in this paper for sixwave mixing realized in Rhodamine $6 \mathrm{G}$ dye are in good agreement with the adopted theoretical model of a resonant medium. Similar results in earlier publications have been obtained for the case of wavefront conjugation upon fourwave mixing ${ }^{4}$.

\section{THEORETICAL MODEL}

The formation of dynamic holograms in resonant media in conditions of higher-order nonlinearities has some salient features. Similar to the classical case, recording of a dynamic hologram is effected by signal $E_{S}$ and reference $E_{1}$

\footnotetext{
*ormachea a bsu.by; Department of Physics, Division of Laser Physics \& Spectroscopy, Belarusian State University, Minsk, 220050. Republic Belarus
} 

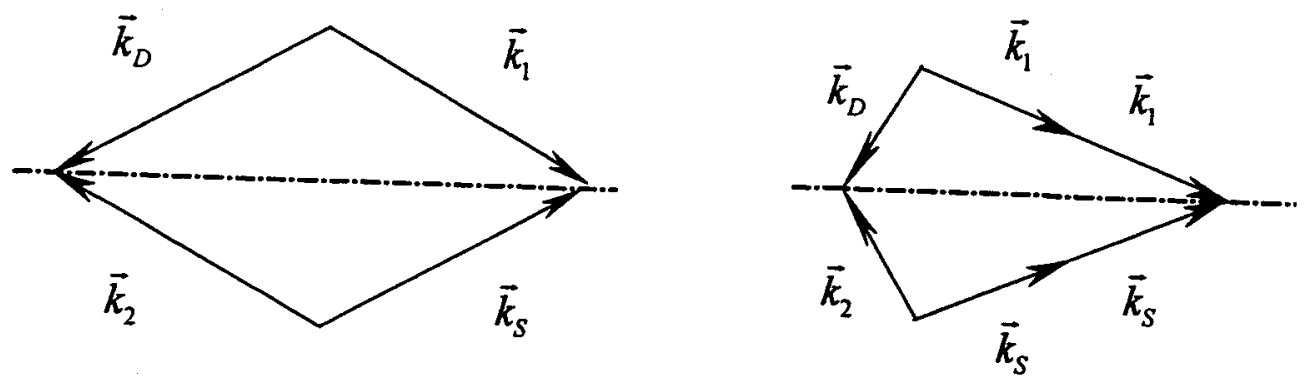

Fig.1. Mixing geometry for four- and six-wave mixing

waves with a frequency $\omega$ (Fig. 1). When the reading wave $E_{2}$ is propagating in counter direction with the same frequency $\omega$ (first-order diffraction), the nonlinear polarization $\mathrm{P}=\chi^{(3)} E_{1} E_{s}^{*} E_{2}$ is induced and we have a traditional variant of wavefront conjugation on four-wave mixing, $i$. e. the diffracted wave $E_{D}$ is precisely counter-propagating relative to wave $E_{S}$ and independent of the signal wave propagation direction.

The condition of higher-order Bragg diffraction may be satisfied through a change in the propagation direction of the reading wave $E_{2}$. As this takes place, the diffracted wave $E_{D}$ is determined by nonlinear polarization $P=\chi^{(N-1)}\left(E_{1} E_{S}^{*}\right)^{M} E_{2}$, where $M$ is the diffraction order and $N$-wave mixing $(N=2(M+1))$ due to the $(N-1)$-order diffraction is the case (Fig. 1). The propagation direction of the diffracted wave $E_{D}$ is dictated by the phase synchronism condition $\vec{k}_{D}=M \vec{k}_{1}-M \vec{k}_{S}+\vec{k}_{2}$ that defines the interaction geometry. In the process the reading wave $E_{2}(\omega)$ should be directed to the nonlinear medium at an angle $\beta=(M \sin (\alpha / 2))-\alpha / 2$ relative to the propagation direction of the reference wave $E_{1}$, where $\alpha$ is an angle between the hologram recording light beams $(\beta \approx(M-1) \alpha / 2$ for small values of $\alpha)$. The same relationship follows for angle $\beta$ from analysis of the Bragg condition in case of diffraction from the $M$-th component of a dynamic grating. Likewise, propagation directions of the
diffracted and signal waves also differ in the $\beta$ angle.

A system of equations describing the formation of a diffracted wave is of the form ${ }^{5}$

$$
\begin{aligned}
& \frac{\partial E_{1, S}}{\partial z}=i \frac{2 \pi \omega}{c n_{0}}\left(E_{1, S} \chi_{0}(\omega)+E_{S, 1} \chi_{ \pm 1}(\omega)\right) \\
& \frac{\partial E_{2, D}}{\partial z}=-i \frac{2 \pi \omega}{c n_{0}}\left(E_{2, D} \chi_{0}(\omega)+E_{D .2} \chi_{ \pm M}(\omega)\right),
\end{aligned}
$$

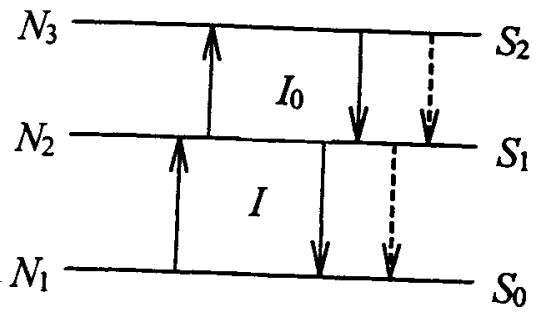

Fig.2. A scheme of optical irradiation $I_{0}$ an multi-wave mixing $I$ realized in three-level medium. susceptibility $\chi$ in harmonics of the dynamic grating $\zeta=\left(\vec{\kappa}_{1}-\vec{\kappa}_{S}\right) \vec{r}$. The terms with $\chi_{0}$ include changes in the absorption coefficient and refractive index of a bleaching medium in the interference field of interacting waves, $\chi_{ \pm 1}$ defines rescattering of the signal and reference waves from the forming phase-amplitude grating, and $\chi_{ \pm M}$ defines the parametric coupling between reading and diffracted waves.

A specific form of the nonlinear susceptibility $\chi$ is dictated by spectroscopic characteristics of the medium and by selection of the excitation frequency. An adequate description of complex organic compounds (dyes) characterized by the overlapping absorption and emission bands from different energy levels necessitates the use of 
multilevel models for the resonant medium. In the present work the light-induced response in conditions of twofrequency excitation has been described by the model based on a three-level scheme of the energy states of dye molecules including transitions between the excited singlet states and absorption of an external optical pumping from the excited level (Fig. 2), the solid curves denote induced transitions, and the dashed lines denote spontaneous and radiationless transitions.

Similar to the case of four-wave mixing ${ }^{6}$, the solution for coupled kinetic equations describing the level populations $\mathrm{N}_{i}$ in the stationary interaction mode may be as follows:

$$
\begin{aligned}
& N_{1}=N\left(1+B_{21} I / v P_{21}\right)\left(1+B_{32} I_{0} / v P_{32}\right) / K, \\
& N_{2}=N\left(B_{12} I / v P_{21}\right)\left(1+B_{32} I_{0} / v P_{32}\right) / K, \\
& N_{3}=N\left(B_{12} B_{23} I I_{0} / v^{2} P_{21} P_{32}\right) / K,
\end{aligned}
$$

where

$$
\begin{gathered}
N=N_{1}+N_{2}+N_{3}, \\
K=1+\left(B_{12}+B_{21}\right) I / v P_{21}+B_{32} I_{0} / v P_{32}+\left(B_{12} B_{23}+B_{12} B_{32}+B_{21} B_{32}\right) I I_{0} / v^{2} P_{21} P_{32} .
\end{gathered}
$$

Here $I, I_{0}$ are radiation intensities in channels $S_{0}-S_{1}$ (waves involved in the process of multiwave mixing) and $S_{1}-S_{2}$ (optical pumping) respectively, $v$ is the velocity of light in the medium, $P_{i j}$ is the probability of spontaneous and nonradiative transitions in $i-j$ channel. The Einstein coefficients for the induced transitions $B_{12}$ and $B_{21}$ are determined at the frequency $\omega$ of radiation $I$, whereas for $B_{23}$ and $B_{32}$ - at the shifted frequency $\omega^{*}$ of optical pumping $I_{0}$.

In calculations for the nonresonance properties of a resonant medium we take into consideration the fact that the complex refractive index due to resonance transitions in the channel $i-j$ is described by the following expression?:

$$
\hat{n}_{i j}=\frac{h c}{2 v}\left(\mathrm{~N}_{i} \hat{\theta}_{i j}(\omega)-\mathrm{N}_{j} \hat{\theta}_{\mu}(\omega)\right)
$$

where $\hat{\theta}_{i j}(\omega)=\theta_{i j}(\omega)+i B_{i j}(\omega), \theta_{i j}(\omega)$ are related by the Kramers-Kronig relations with Einstein's coefficients $B_{i j}(\omega)$. Thermal change of the refractive index in conditions of adiabatic heating of the medium is of the form[7]:

$$
\Delta n_{T}=\frac{h c}{2 v}\left(\sigma I\left(N_{1} B_{12}-N_{2} B_{21}\right)\left(1-\mu_{21}\right)+\sigma^{\cdot} I_{0}\left(N_{2} B_{23}-N_{3} B_{32}\right)\left(1-\mu_{32}\right)\right)
$$

where $\sigma_{\mathrm{T}}=2 \omega(d n / d \mathrm{~T}) \tau / c C_{\mathrm{\rho}}$ at the frequency $\omega$ and $\sigma^{\circ} \mathrm{T}=2 \omega^{\circ}(d n / d \mathrm{~T}) \tau / c C_{\mathrm{p}}$ at the frequency $\omega^{\circ}, \tau$ is the interaction duration, $C_{\rho}$ is the unit volume heat capacity, $\mu_{g}$ - quantum yield of luminescence in $i-j$ channel, and $d n / d \mathrm{~T}$ - thermooptic coefficient.

Taking into consideration the relationships (3) - (5), the nonlinear medium susceptibility may be given as

$$
\chi_{n u}=\frac{n_{0} \kappa_{0}}{2 \pi}\left(\frac{\hat{\theta}_{\mathrm{i} z}}{B_{12}}-\frac{\hat{\alpha}\left(\omega, \omega^{-}\right) I}{1+\alpha I}\right)
$$

where $I=I_{1}+I_{2}+I_{S}+2 \sqrt{I_{1} I_{S}} \cos \left(\left(\vec{k}_{1}-\vec{k}_{S}\right)+\varphi-\emptyset_{s}\right)$ is the interference field intensity of the waves recording the dynamic-grating $I_{1}, I_{S}$ in presence of the reading wave $I_{\text {: }}$

$$
\hat{\alpha}=a+i \alpha=\frac{\hat{\theta}_{12}(\omega)+\hat{\theta}_{21}(\omega)}{v P_{21}}-\sigma\left(1-\mu_{21}\right)-\frac{B_{23} I_{0}\left(\hat{\theta}_{12}(\omega) / v P_{32}-\sigma^{*}\left(1-\mu_{32}\right)\right)}{\left(1+B_{32} I_{0} / v P_{32}\right) v P_{21}}
$$

Relation (6) enables one to calculate the Fourier expansion components for $\chi_{ \pm M}$, which are involved in a system of equations (1), (2)

$$
\chi_{0}(\omega)=\frac{n_{0} \aleph_{0}}{2 \pi}\left[\frac{\hat{\theta}_{12}}{B_{12}}-\frac{\hat{\alpha}}{\alpha}+\frac{(\hat{\alpha} / \alpha)}{A_{0}}\right]
$$




$$
\begin{aligned}
\chi_{ \pm 1}(\omega) & =\frac{n_{0} \aleph_{0}}{2 \pi}\left[\frac{2 \hat{\alpha} \sqrt{I_{1} I_{S}}}{A_{0}\left(1+\alpha I+A_{0}\right)}\right] e\left[ \pm i\left(\varphi_{1}-\varphi_{S}\right)\right] \\
\chi_{ \pm M}(\omega) & =\frac{n_{0} \aleph_{0}}{2 \pi}\left(\frac{\hat{\alpha}}{\alpha}\right) \frac{\left(-2 J \sqrt{I_{1} I_{S}}\right)^{M}}{A_{0}\left(1+\alpha I+A_{0}\right)^{M}} e\left[ \pm i M\left(\varphi_{1}-\varphi_{S}\right)\right]
\end{aligned}
$$

It should be noted that with due regard for expressions (8) - (10), the parameters (intensity and frequency) of incoherent optical pumping are directly involved in coupled equations (1), (2) through the complex coefficient $\bar{\alpha}$ (7).

The results of numerical analysis for the system of equations (1), (2) describing the multiwave mixing are illustrated in Fig. 3. The diffraction efficiency $\xi=I_{D}(r=0) / I_{2}(r=L)$ has been calculated for a three-level medium model in the assumption of the dye excitation into the center of absorption bands $S_{0}-S_{1}$ (waves involved in recording of dynamic holograms) and $S_{1}-S_{2}$ (optical pumping). Along with resonance nonlineraity, thermal nonliearity has been included for the following parameters of medium and radiation: pulse length $\tau=10 \mathrm{~ns}$, refractive index $n_{0}=1.36$, thermooptic
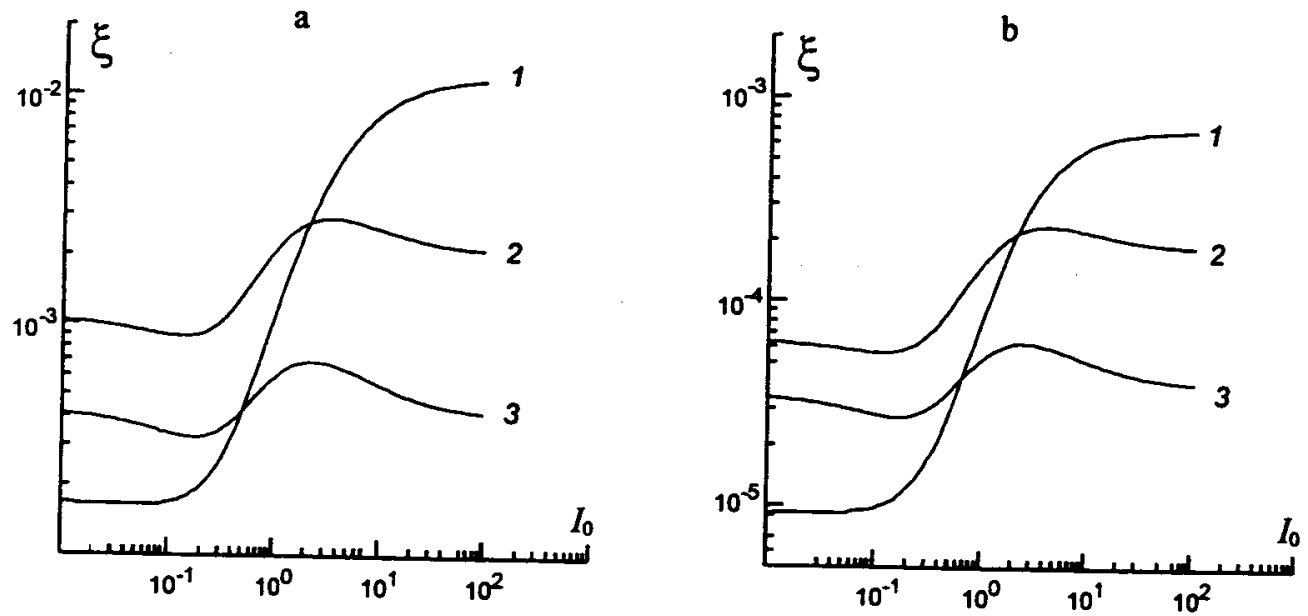

Fig.3. The diffraction efficiency for a) four-, and b) six-wave mixing as a function of the irradiation intensity when $I_{p}=0.1(1), 0.8(2), 2$ (3).

coefficient $(d n / d T) C_{p}^{-1}=-10^{-4} \mathrm{~J}^{-1} \mathrm{~cm}^{3}$, quantum yield of luminescence $\mu_{21}=0.5, \mu_{32}=\mu_{21} / 800$, initial optical density $k_{0} L=1$, and spectral characteristics of the medium: $2 \lambda_{12}=\lambda_{23}=1 \mu m, 4 \Delta \lambda_{12}=\Delta \lambda_{23}=100 \mathrm{~nm}(\lambda$ and $\Delta \lambda$ are respectively the center and halfwidth of the absorption band). The above parameters conform to the singlepulse excitation conditions for dye solutions in ethanol.

As seen, optical pumping in the excited channel allows for a considerable (by several orders of magnitude) increase in the energy efficiency of multiwave mixing. Analysis of the terms in the expression for $\bar{\alpha}$ (7) reveals the predominance of thermal nonlinearity due to thermalization of the absorbed energy in the excited channel. Proceeding from the nonuniform population of the level $S_{1}$ (according to interference of the signal and reference waves), the optical pumping energy absorbed in the excited channel is also space modulated. This results in the spatially inhomogeneous thermal release and hence recording of the additional thermally-induced interference structures within the medium volume, which are also involved in the process of multiwave mixing. In consequence of low quantum yield of luminescence in $S_{1}-S_{2}$ channel, the induced thermal gratings are very effective and provide for a significant improvement in the interaction efficiency. The greatest magnification of the dynamic holograms recorded in the principal channel is achieved with relatively low intensities of the hologram recording waves (curve 1), being associated with distortions of the groove profile on saturation of the resonance transition $S_{0}-S_{1}$. Some decrease in the diffraction efficiency at low pumping intensities $\left(\alpha_{0} I_{0} \sim 5\right)$ is due to differing signs of the resonance (at a frequency of interaction) 
and thermal nonlinearities. With their equal absolute values, the formation of a diffracted wave is conditioned by diffraction from the amplitude dynamic gratings only.

Note that optical pumping is responsible for magnification of the recorded hologram owing to the transitions from the excited level, practically without effect for the process of holographic recording in the principal channel. As this takes place, one is enabled to realize magnification of a dynamic grating in the linear mode using very high pumping intensities, including the saturation intensities of the resonance transition between the excited energy states of molecules.

\section{EXPERIMENTAL REALIZATION OF THE MULTIWAVE MIXING ENHANCEMENT BY INDEPENDENT OPTICAL PUMPING}

Applicability and efficiency of the method proposed for the energy efficiency enhancement of multiwave mixing using additional incoherent optical pumping have been demonstrated with Rhodamine $6 \mathrm{G}$ dye solution in ethanol as a resonant medium modeled by a three-level scheme of the molecular energy states.

It should be noted that in earlier works the magnification effect of faint light-induced gratings recorded in a single spectral channel through nonlinearity of other channel has been supported by the experimental results during realization of wavefront conversion on four-wave mixing ${ }^{4,6}$. A threefold increase in the reflection coefficient of a return wave has been attained with the additional pumping intensity increase up to $250 \mathrm{MW} / \mathrm{cm}^{2}$ at the fundamental lasing frequency of an yttrium aluminum garnet laser. In the process, additional pumping radiation at a wavelength of $\lambda=1.064 \mu \mathrm{m}$ was not optimal for a significant enhancement of the return wave since the absorption cross-section from the excited level at the additional pumping frequency was one twentieth of that from the ground level for the dynamic-hologram recording waves at $\lambda=532 \mathrm{~nm}$. To lower the additional pumping intensity, in the present work it has been proposed to use another wavelength of additional optical pumping for which the absorption section from the excited level was comparable to that of the molecules in the ground state.

Fig. 4 presents the experimental setup. Recording and reconstruction of a dynamic hologram was realized by second-harmonic radiation of yttrium aluminum gamet laser $(\lambda=532 \mathrm{~nm}, \tau=20 \mathrm{~ns})$ falling within the absorption band

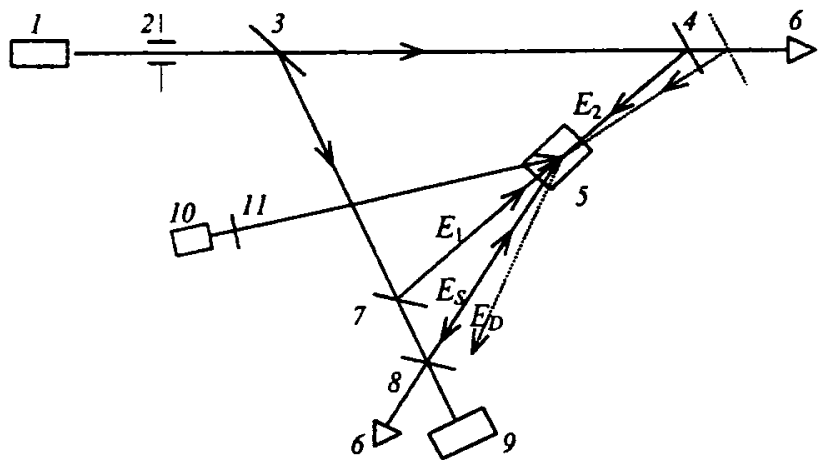

Fig.4. The experimental setup

$S_{0}-S_{1}$ of Rhodamine $6 \mathrm{G}$ dye. A spatially-homogeneous portion of the radiation was cut out by a diaphragm (2) $2 \mathrm{~mm}$ in diameter. Mirrors (3,7 and 8 ) formed signal and reference waves. A reading wave was directed to the nonlinear medium by mirror 4 . This mirror was movable enabling variation of the reading angle. The angle $\alpha \approx 90 \mathrm{mrad}$ between the propagation directions of the reference and signal beams made possible the overlapping of interacting waves over the whole length of the dye cell $(L=0.45 \mathrm{~cm}$ ). Single-pulse radiation of a similar dye (Rhodamine $6 \mathrm{G}$ ) laser at a wavelength of $\lambda=570 \mathrm{~nm}$ was used as additional incoherent pumping in $S_{1}-S_{2}$ absorption band. Actually, the dye laser radiation $(10)$ was directed to the nonlinear medium concurrently with the reference waves and signal beam. Filters $(11)$ enabled one to change the intensity of an additional light beam with invariable intensities of the references and signal waves. The energy efficiency of multiwave mixing $\xi=I_{D} / I_{2}$ (intensity ratio of the diffracted $I_{D}$ and reading $I_{2}$ beams) was measured by the system 6,9 .

The relationship between the diffraction efficiency and the additional optical pumping intensity at the entrance to the dye cell obtained for different values of the dye solution optical density are given in Fig. 5 . As seen, by the use of additional optical pumping the efficiency of four- and six-wave mixing has been increased by three- and sevenfold. As 
this took place, the additional pumping intensity was $2-3 \mathrm{MW} / \mathrm{cm}^{2}$ only, being less by two orders of magnitude than the values cited in ${ }^{4,6}$ for the case of four-wave mixing.
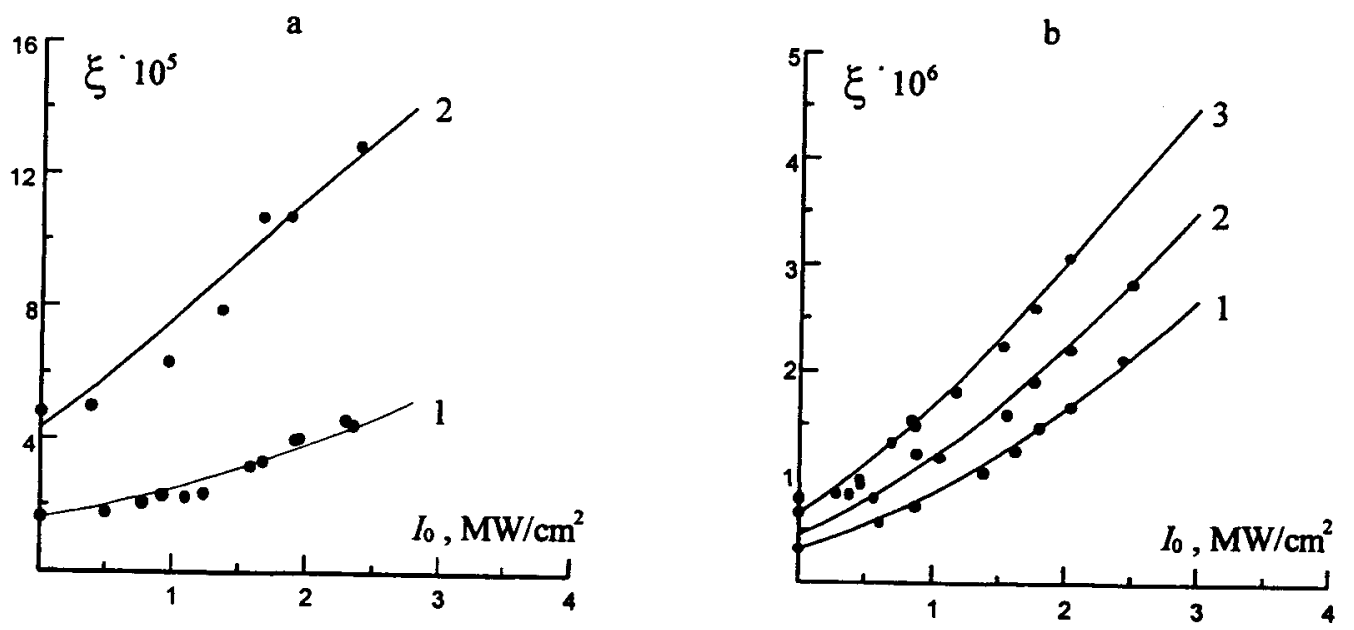

Fig.5. The diffraction efficiency as a function of the irradiation intensity $I_{0}$, for a) four- $k_{0} L=1.7$ (1), 4.3 (2) and b) six-wave mixing $k_{0} L=2.9$ (1), 3.4 (2), 4 (3)

\section{CONCLUSION}

Thus, the performed theoretical and experimental studies of multiwave mixing in solutions of complex organic compounds have demonstrated that additional irradiation of the medium at a frequency associated with transitions between the excited energy levels holds much promise. Absorption of additional optical pumping in the excited channel results in recording of extra thermally-induced interference gratings within the medium volume which are involved in the process of multiwave mixing and are responsible for the enhanced diffraction efficiency of dynamic holograms. The considered scheme for the multiwave-mixing enhancement has advantage of additional optical pumping that induces additional dynamic gratings without very high coherence and monochromaticity, as for the references fields. Optimization of the frequency of additional optical pumping makes it possible to achieve a considerable magnification at the pumping intensities about several $\mathrm{MW} / \mathrm{cm}^{2}$.

\section{REFERENCES}

I. J.W.R. Tabosa, C.L. Cesar, M. Ducloy, J.R Rios Leite, "Degenerate multiwave mixing in SF 6 " Optics Communications. 67, pp. 240-244, 1988.

2. A. Blouin., M-M. Denariez-Roberge, P. Galarneau, "Degenerate N-wave mixing in a saturable absorber," Opt. Soc. Am. B. 8, pp. 578-583, 1991.

3. A.S. Rubanov, A.L. Tolstik, S.M. Karpuk, O. Ormachea, "Nonlinear formation of dynamic holograms and multiwave mixing in resonant media," Optics Communications. 181, pp. 183-190, 2000.

4. E.V. Ivakin, S.M. Karpuk, A.S. Rubanov, A.L. Tolstik, A.V. Chaley, "Incoherent amplification of phase conjugation in solutions of dyes," Technical Physics Letters. 17, N 14, pp. 56-59, 1991.

5. A.L. Tolstik, "Wave front transformation by nonlinear formed dynamic holograms," Proceedings SPIE. 3684, pp.

6. S.M. Karpuk, A.S. Rubanov, A.L. Tolstik, A.V. Chaley, "Four-wave mixing in multilevel media at spatially homogeneous irradiation," Proceedings SPIE. 1840, pp. 43-51, 1992.

7. V.V. Kabanov, A.S. Rubanov, A.L. Tolstik, "An influence of transitions between excited singlet and triplet states upon phase response of dye solutions," Kvant. Electron. 15, pp. 1681-1686, 1988, in Russian; Sov. J. Quantum Electron. 18, pp. 1047-1052, 1988. 